\title{
Perception of Combined Visual and Inertial Low-Frequency Yaw Motion
}

\author{
Ana Rita Valente Pais;, M. M. (René) van Paassen ${ }^{\dagger}$ and Max Mulder \\ Delft University of Technology, Delft, The Netherlands \\ and \\ Mark Wentink ${ }^{\S}$ \\ TNO Defence, Security and Safety, Soesterberg, The Netherlands
}

\begin{abstract}
Following a previous study in the Simona simulator on perception of coherent visual and inertial cues in a flight simulator, an experiment is performed in the Desdemona simulator to investigate the influence of the frequency of visual and inertial stimuli on the limits of the perceived coherence zone. The coherence zone is defined as the range of inertial motion amplitudes that, though not being a physical match to the visual cues, are still perceived by subjects as coherent. The main hypothesis tested is that the semi-circular canals dynamics influence the internal comparison between the visually and inertially perceived self-velocity. Furthermore, the results between the Simona and Desdemona studies are compared. In general, the effect of amplitude and frequency on the measured coherence zones follow the same trends as in the previous study: the coherence zone width increases with increasing visual cue amplitude and the point of mean coherence decreases with respect to the oneto-one line for the higher amplitudes. The results for the low frequency and low amplitude stimulus might be affected by the inertial sensory threshold, making it difficult to draw definite conclusions about the posed hypothesis. The results between the two simulator studies are different in terms of absolute values, but the trends are the same.
\end{abstract}

\section{Introduction}

In a flight simulator the pilot is subject to inertial and visual cues that do not always physically match. Whereas the simulator inertial motion is determined by the motion filters, the visual cues represent the actual aircraft motion. Inertial and visual cues might differ up to a certain extent without any consequences for the simulation quality. However, if the difference becomes large enough to be perceived by the pilot in the simulator, then the simulation realism is impaired. ${ }^{1}$ For this reason, research on human self-motion perception during combined visual and inertial stimulation is of crucial importance for flight simulation.

Many have studied the influence of combined visual and inertial cues in self-motion perception ${ }^{2-13}$ and the maximum mismatch between visual and inertial cues that is still considered acceptable. ${ }^{14-18}$

Van der Steen ${ }^{1}$ introduced the term coherence zone to refer to the range of inertial motion amplitudes that although not physically matching the visual motion cues, are still perceived by subjects in a simulator as being congruent.

In a previous study on yaw perception coherence zones, ${ }^{18}$ it has been shown that at high frequencies subjects prefer inertial motion amplitudes that are lower than the physically correspondent visual cue amplitudes. This finding was explained using the dynamics of the Semi-Circular Canals (SCC): at higher

*Ph.D. student, Faculty of Aerospace Engineering, Control and Simulation Division, A.R.ValentePais@tudelft.nl, P.O. Box 5058, 2600 GB Delft, The Netherlands, AIAA Student Member.

${ }^{\dagger}$ Associate Professor, Faculty of Aerospace Engineering, Control and Simulation Division, M.M.vanPaassen@tudelft.nl, P.O. Box 5058, 2600 GB Delft,The Netherlands, AIAA Member.

$\ddagger$ Professor, Faculty of Aerospace Engineering, Control and Simulation Division, M.Mulder@tudelft.nl, P.O. Box 5058, 2600 GB Delft, The Netherlands, Senior AIAA Member.

§Researcher, mark.wentink@tno.nl, P.O. Box 23, 3769 ZG Soesterberg, The Netherlands. 
frequencies, above $9 \mathrm{rad} / \mathrm{s}$ the velocity signal is more amplified than at the middle frequencies (from 1 to $9 \mathrm{rad} / \mathrm{s})$. At higher frequencies a higher gain is introduced in the vestibular sensory path but not in the visual path. When the visual and vestibular signals are compared internally, the vestibular signal will be perceived as having higher amplitude than the visual signal. When asked to match the amplitude of the inertial signal with that of the visual, subjects will then tend to down tune the inertial signal. Although this line of reasoning seems to explain the previously obtained results, it depends on the assumption that the dynamics of the SCC are not being taken into account during the internal comparison of the visual and vestibular signals in the Central Nervous System (CNS). This implies that there is no internal representation of the SCC dynamics or that this representation is faulty.

The present paper further investigates the assumption of the imperfect internal model by measuring yaw coherence zones at low frequencies, in this case at $0.2 \mathrm{rad} / \mathrm{s}$, and comparing it to measurements at $2 \mathrm{rad} / \mathrm{s}$. The results are analyzed in velocity and acceleration space.

This paper is organized as follows: in the next section the hypothesis posed is explained in more detail, followed by the experimental method with a detailed description of the choices made during the experimental design. Afterward, the results are presented and compared with those from the previous study on yaw coherence zones at higher frequencies. At the end, results are discussed and conclusions drawn.

\section{Hypothesis}

Based on previous experiments ${ }^{18,19}$ the hypothesis is made that the dynamics of the SCC are not taken into account in the internal comparison between perceived visual and perceived inertial motion, which implies that the perceived coherence zones will vary depending on the frequency of the stimuli used. For a more concrete idea of this dependency, the dynamics of the SCC are plotted in Figure 1 together with an indication of the motion stimuli frequency used in this experiment (black circles).

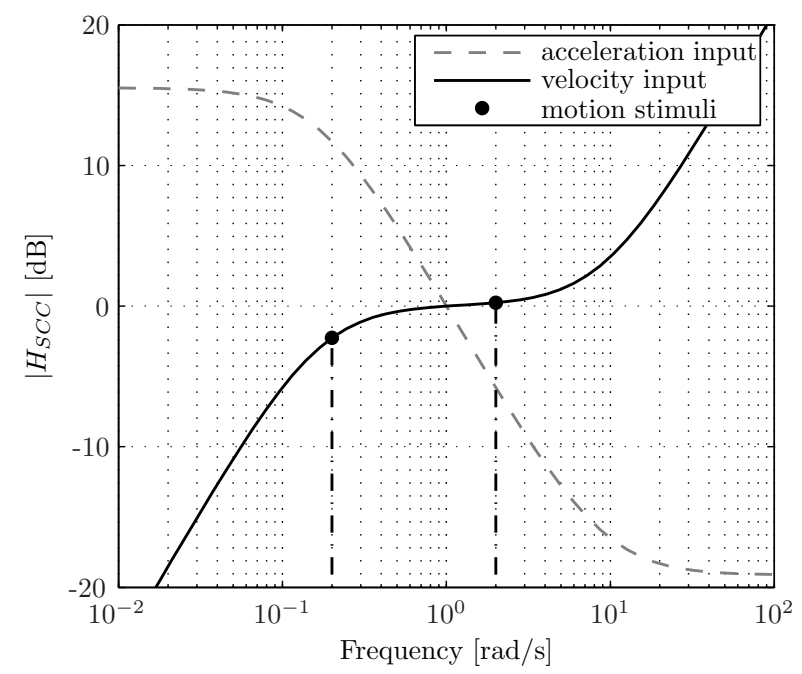

Figure 1. Semi-circular canals dynamics represented in terms of acceleration and velocity input and motion stimuli frequency.

The displayed dynamics are based on the model fitted by Hosman and van der Vaart ${ }^{20}$ and scaled such that at the frequency of $1 \mathrm{rad} / \mathrm{s}$ the gain equals one. Equation (1) represents the dynamics of the SCC when considering a velocity input.

$$
H_{S C C}(j \omega)=5.972 j \omega \frac{0.1097 j \omega+1}{5.924 j \omega+1}
$$

For a frequency range between 1 and $9 \mathrm{rad} / \mathrm{s}$ the SCC dynamics equal approximately a gain of one. For frequencies higher than $9 \mathrm{rad} / \mathrm{s}$ the SCC introduce a gain higher than one in the vestibular path signal. If the visual and vestibular signals are directly compared and there is no internal compensation for the gain introduced by the SCC dynamics, then the vestibularly perceived motion amplitude will be higher than the visually perceived motion amplitude. As a result of this perceived difference, subjects will require lower amplitudes of inertial motion than the amplitude that physically corresponds with the visual cue. This 
reasoning is supported by the results of a previous study that measured yaw coherence zones for frequencies of 2 and $10 \mathrm{rad} / \mathrm{s}^{18}$ For the lower frequency range the opposite should happen. For the chosen frequency of $0.2 \mathrm{rad} / \mathrm{s}$ the gain introduced by the SCC is lower than one so, the hypothesis is posed that subjects should prefer inertial motion amplitudes that are higher than the one-to-one match with the visual stimulus. This would imply that there is no internal model of the SCC dynamics or that this model is not accurate for too high or too low frequencies.

\section{Method}

\section{III..1. Apparatus}

The experiment was conducted in the Desdemona simulator at TNO Defence, Security and Safety in Soesterberg, The Netherlands.

Figure 2 is a schematic of Desdemona with indication of its 6 degrees of freedom. Table 1 summarises the Desdemona motion space specifications. The simulator has an 8 meter linear track that can rotate around its central point, providing a 4 meter centrifuge arm. The structure mounted on the linear track consists of a 2 meter vertical linear track. A gimballed structure mounted on the heave track allows the cabin to rotate more than 360 degrees in three orthogonal axes. Using common aeronautical nomenclature we name the rotations around the vertical axis "cabin yaw". In this experiment only the cabin yaw was used.

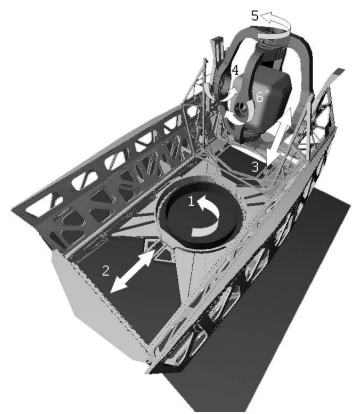

Figure 2. Artistic impression of the Desdemona simulator with indication of the degrees of freedom: 1. Central yaw axis. 2. Radius track. 3. Heave track. 4. Cabin roll. 5. Cabin yaw. 6. Cabin pitch.

Table 1. Cabin yaw motion space limits.

\begin{tabular}{lc}
\hline \hline & Cabin \\
& yaw \\
\hline Position & $>360 \mathrm{deg}$ \\
Velocity & $180 \mathrm{deg} / \mathrm{s}$ \\
Acceleration & $90 \mathrm{deg} / \mathrm{s}^{2}$ \\
\hline \hline
\end{tabular}

A PC-based computer generated image system was used to render the outside world. In the cabin, three computers generated real-time images with an update rate of $60 \mathrm{~Hz}$. Three projectors (resolution: $1024 \times$ $768 \mathrm{px}$ ) projected the image on a three part flat screen, placed at approximately $1.5 \mathrm{~m}$ from the participants' eyes, creating an out-of-the-window field-of-view of 120 degrees horizontal and 32 degrees vertical. The outside visual scene consisted of a view of an airport including some buildings, part of a runway and some grass fields. The viewpoint height was 5 meters.

\section{III..2. Experimental design}

To measure coherence zones in yaw with a low frequency stimulus, a very large position motion space is necessary which is why the experiment was performed in the Desdemona simulator. However, it would be very interesting to compare the results of the present experiment with the ones from the previous study ${ }^{18}$ which was performed in the Simona Research Simulator, an hexapod-like simulator. For this reason, some experimental conditions were chosen to match exactly the ones also used in that study. So, as in the Simona study, two experimental conditions were defined by two visual amplitudes: 12 and $30 \mathrm{deg} / \mathrm{s}^{2}$, and one stimuli frequency: $2 \mathrm{rad} / \mathrm{s}$.

Furthermore, to investigate the effect of frequency on the coherence zones in both velocity and acceleration space a combination of frequency and amplitude of the stimuli had to be found that allowed for a comparison between signals with two different frequencies and the same acceleration amplitude and two different frequencies and the same velocity amplitude. For the low frequency stimulus a frequency of 0.2 $\mathrm{rad} / \mathrm{s}$ was chosen. Since due to simulator velocity limits it was not possible to perform the condition defined by amplitude $30 \mathrm{deg} / \mathrm{s}^{2}$ and frequency $0.2 \mathrm{rad} / \mathrm{s}$, one other amplitude value was defined: $3 \mathrm{deg} / \mathrm{s}^{2}$. Using this 
amplitude at a frequency of $0.2 \mathrm{rad} / \mathrm{s}$ allowed for a direct comparison, in velocity space, with the condition with visual amplitude of $30 \mathrm{deg} / \mathrm{s}^{2}$ and frequency of $2 \mathrm{rad} / \mathrm{s}$, since both result in a velocity amplitude of 15 $\operatorname{deg} / \mathrm{s}$.

The visual scene was also chosen to provide similar references as in the Simona study. However, since the visual data bases used were different and the outside scene was not exactly the same, it was thought that some investigation into the effect of the content of the visual scene would be interesting as well. For this reason and with an exploratory character, a condition was added with a different visual scene consisting of a star field. At an amplitude of $12 \mathrm{deg} / \mathrm{s}^{2}$ and a frequency of $2 \mathrm{rad} / \mathrm{s}$, measurements were made under two different outside visual conditions: the airport scene and the star field, which consisted of white dots on a black background. In this way only visual flow was provided and there was no other elements that could give a real measure of distance or attitude. The lack of concrete references is thought to influence subject's estimate of visually perceived attitude. ${ }^{21}$

Table 2 shows a summary of the visual motion conditions. Note that the condition with amplitude of 12 $\mathrm{deg} / \mathrm{s}^{2}$ and frequency of $2 \mathrm{rad} / \mathrm{s}$ was tested with both the airport scene and the star field.

Table 2. Experimental conditions defined in terms of acceleration amplitude and frequency of the visual stimuli and the resulting visual amplitude in $\mathrm{deg} / \mathrm{s}$.

\begin{tabular}{ccccc}
\hline \hline & & \multicolumn{3}{c}{ Amplitude, deg/s } \\
\cline { 2 - 5 } & & 3 & 12 & 30 \\
\hline Frequency, & 0.2 & 15 & 60 & - \\
$\mathrm{rad} / \mathrm{s}$ & 2 & 1.5 & 6 & 15 \\
\hline \hline
\end{tabular}

In total there were six different experimental conditions. For each condition both upper and lower thresholds were measured and each subject repeated each measurement 3 times which resulted in a total of 36 trials per subject.

\section{III..3. Procedure}

Thresholds were measured using an in-the-loop self-tuning procedure also used in reference 22 . While being subject to a continuous sinusoidal yaw motion both visually and inertially, subjects were asked to use a joystick to change the inertial motion gain.

The joystick gain was different for the two frequencies and it was chosen as a compromise between control sensitivity and tuning procedure duration. A high joystick gain would cause abrupt or fast changes in the inertial motion amplitude, possibly resulting in discomfort or making it very difficult for subjects to finetune the motion gain. A too low gain would result in very long experimental trials. The joystick gain was set such that at the maximum deflection, either positive or negative, the joystick signal would cause the velocity signal amplitude to double in approximately $84 \%$ of the stimulus period. The joystick signal was integrated and to further smooth the transition between inertial motion gains a second-order low-pass filter $\left(\omega_{n}=5, \xi=1\right)$ was applied. The output of the second-order filter was then applied to the inertial velocity signal. Additionally, some logic was applied to the motion gain signal to prevent negative gains or too high gains that would cause the simulator to reach its motion limits.

For measurements of the upper threshold, participants were asked to increase the motion gain as much as possible while still perceiving the inertial motion amplitude to match the visually perceived motion. For measurements of the lower threshold, they were asked to decrease the motion gain as much as possible while still perceiving matching inertial and visual motion amplitudes. When the desired motion gain was achieved, subjects pressed the trigger button on the joystick to end the trial. A second press on the trigger indicated they were ready for the next trial. The initial motion gain was a random value between 1.15 and 0.85 .

\section{III..4. Subjects and subjects' instructions}

Seven male subjects and one female subject participated in the experiment. Their ages were between 21 and 28 years, with an average of 24.63 years.

Participants were seated and held the joystick in their right hand. They were asked to keep their head as much as possible in the seat's head-rest and refrain from making head movements, but they were free to scan the outside view at will. They wore a head-set where white noise was played to mask simulator noise. The head-set also allowed them to communicate with the experiment supervisor at all times. 
Subjects were instructed to make small adjustments to the motion gain and after every adjustment let the motion profile run for at least one full period. They were also advised to adjust the motion gain until they were outside their coherence zone, that is, while measuring an upper threshold they should increase the motion gain until they felt the inertial motion was too strong. From that point they should slowly decrease the motion gain until it felt congruent again.

\section{Results}

Threshold values were obtained by computing the maximum inertial motion amplitude during the last period of the sinusoidal signal in each trial. From the upper and lower threshold values a coherence zone width $(\mathrm{CZW})$ and a point of mean coherence (PMC) were calculated using Equation (2) and Equation (3), respectively.

$$
\begin{gathered}
C Z W=t h_{u p}-t h_{l o} \\
P M C=t h_{l o}+\frac{C Z W}{2}
\end{gathered}
$$

Figure 3 displays the PMC and CZW for two visual amplitudes and two frequencies. Values are presented in acceleration.

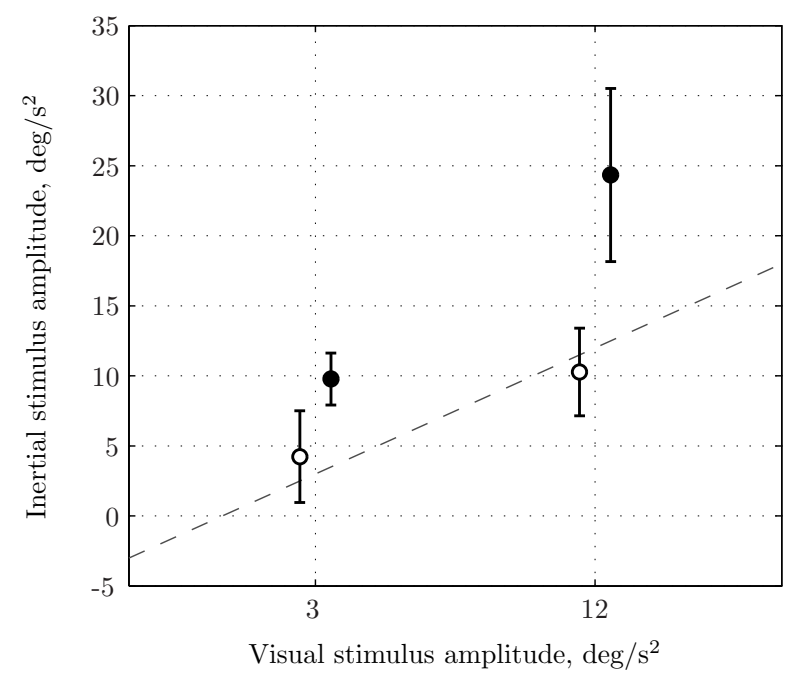

(a) Point of mean coherence.

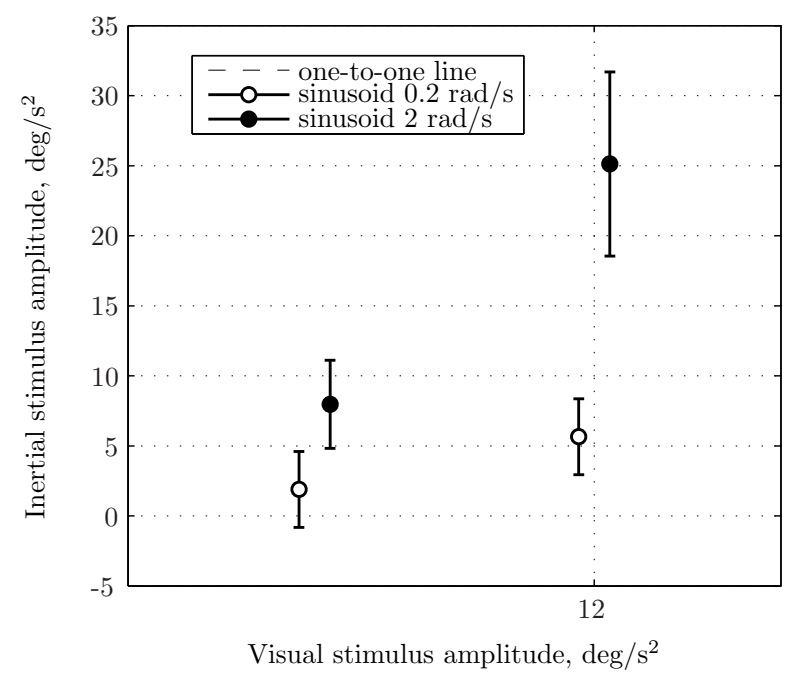

(b) Coherence zone width.

Figure 3. Measured coherence zones for two visual amplitudes and two stimuli frequencies. Error bars indicate the $95 \%$ confidence interval of the adjusted means.

An ANOVA was performed on the effect of the amplitude of the visual cue and the stimuli frequency on the PMC and CZW. The results are displayed in Table 3. Similar to previous experiments, both amplitude and frequency had a significant effect on the PMC and CZW. However, a significant interaction between frequency and amplitude was also found. At the amplitude of $12 \mathrm{deg} / \mathrm{s}^{2}$ and frequency of $2 \mathrm{rad} / \mathrm{s}$ the $\mathrm{PMC}$ increases with respect to the one-to-one line, instead of slightly decreasing, as observed for the lower frequency. The same trend can be observed for the CZW.

Table 3. ANOVA results for the point of mean coherence (PMC) and the coherence zone width (CZW), where ** is highly significant $(p<0.01),{ }^{*}$ is significant $(0.01 \leq p<0.05)$, and - is not significant $(p \geq 0.05)$.

\begin{tabular}{lcccccc}
\hline Independent variables & \multicolumn{4}{c}{ Dependent measures } \\
\cline { 2 - 7 } & df & F & sig. & df & F & sig. \\
\hline Factor & 1,7 & 33.13 & $* *$ & 1,7 & 36.26 & $* *$ \\
\hline Amplitude & 1,7 & 15.50 & $* *$ & 1,7 & 33.80 & $* *$ \\
Frequency & 1,7 & 10.73 & $*$ & 1,7 & 10.46 & $*$ \\
Amplitude $\times$ Frequency & 1,7 \\
\hline \hline
\end{tabular}


From Figure 3 it can also be observed that the PMCs with the lower frequency stimulus were not larger than the PMCs with the higher frequency as it was expected. However, if the same results are respresented in velocity space, as shown in Figure 4, the reverse situation is observed. Here only the two conditions with a velocity amplitude of $15 \mathrm{deg} / \mathrm{s}$ are shown.

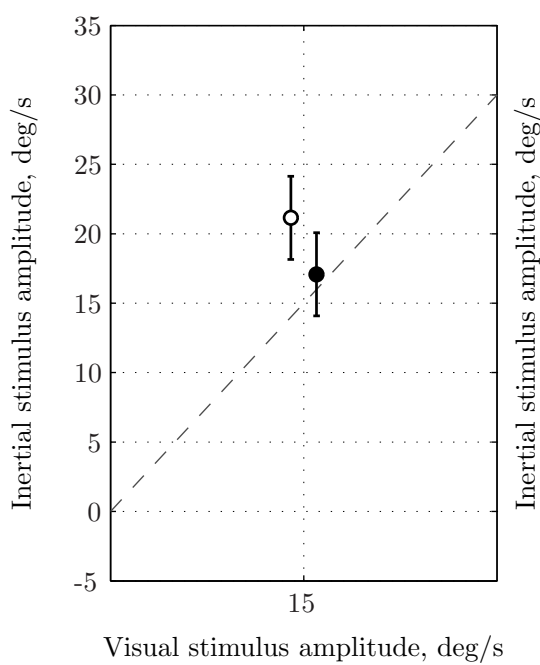

(a) Point of mean coherence.

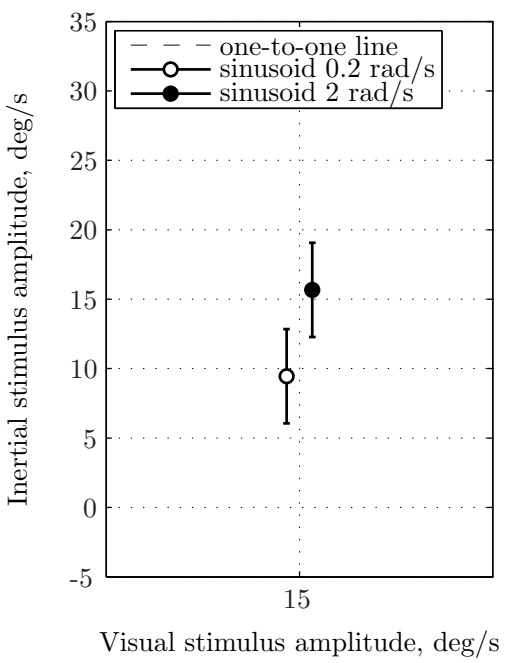

(b) Coherence zone width.

Figure 4. Measured coherence zones for two conditions with the same velocity amplitude and different frequencies. Error bars indicate the $95 \%$ confidence interval of the adjusted means.

Although the PMC with the lower frequency stimulus is higher than with the higher frequency stimulus, comparison using a T-test showed no significant difference between both conditions, $t(7)=1.605, p>0.05$. The CZW values show the inverse trend, with the higher frequency condition having a larger CZW than the lower frequency condition. However, also here the T-test showed that the difference is not significant, $t(7)=-2.166, p>0.05$.

Figure 5 displays the PMCs and CZWs for the two conditions with different outside visuals.

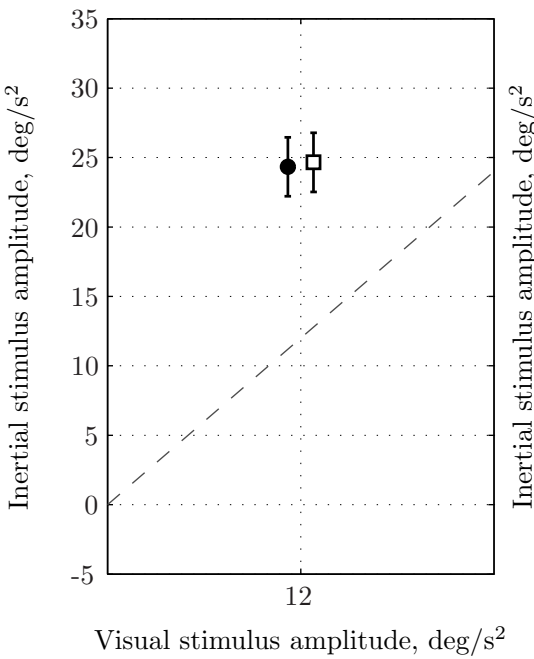

(a) Point of mean coherence.

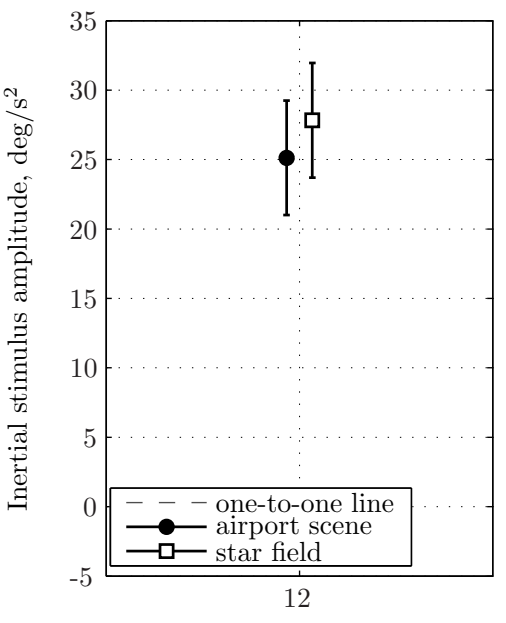

Visual stimulus amplitude, $\mathrm{deg} / \mathrm{s}^{2}$

(b) Coherence zone width.

Figure 5. Measured coherence zones for stimuli frequency of $2 \mathrm{rad} / \mathrm{s}$ and two different outside visual scenes. Error bars indicate the $95 \%$ confidence interval of the adjusted means.

The PMC and CZW for the two visual scenes were fairly similar and the T-tests did not indicate a significant difference between the two conditions: $t(7)=-0.178, p>0.05$ for the $\mathrm{PMC}$ and $t(7)=-0.776, p>0.05$ for the CZW.

The conditions with visual cue amplitude of 12 and $30 \mathrm{deg} / \mathrm{s}^{2}$ and frequency of $2 \mathrm{rad} / \mathrm{s}$ were compared to the ones from the previous study in the Simona simulator. In the latter, the same stimuli characteristics 
were used, although a slightly different tuning method was used and, as mentioned before, different visual display characteristics. Figure 6 shows the means and 95\% confidence interval of the PMC and CZW for the experimental results in the two simulators, Desdemona and Simona.

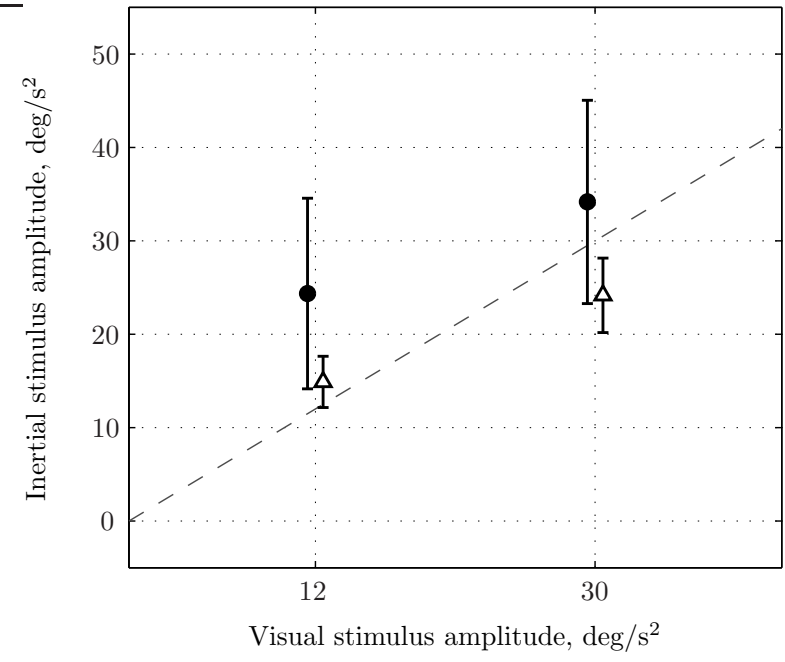

(a) Point of mean coherence.

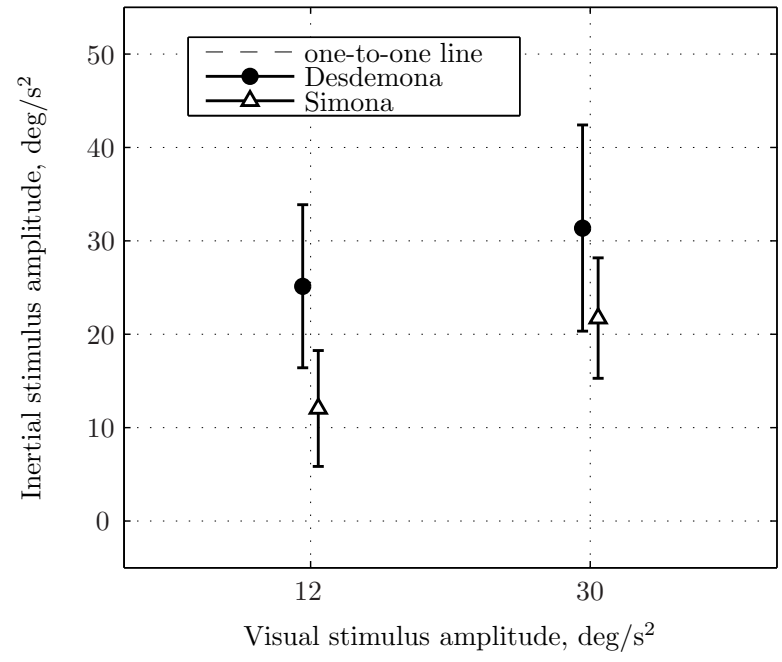

(b) Coherence zone width.

Figure 6. Measured coherence zones for a stimuli frequency of $2 \mathrm{rad} / \mathrm{s}$ and two visual amplitudes in two different experiments in the Desdemona and the Simona simulators. Error bars indicate the 95\% confidence interval of the mean.

The differences between results on both simulators are quite large with the PMC and CZW measured in Simona lower than the ones measured in Desdemona. Also, the 95\% confidence intervals are considerably larger for the experiment in Desdemona. Table 4 shows the results of the ANOVA, where the amplitude of the visual cues was considered a within-subjects effect and the simulator used as a between-subjects effect. To guarantee homogeneity of variance, the PMC data were transformed to the reciprocal and the ANOVA was performed on the transformed data.

Table 4. ANOVA results for the point of mean coherence (PMC) and the coherence zone width (CZW), where ** is highly significant $(p<0.01),{ }^{*}$ is significant $(0.01 \leq p<0.05)$, and - is not significant $(p \geq 0.05)$.

\begin{tabular}{lrrrrrrc}
\hline \hline \multirow{2}{*}{ Independent variables } & \multicolumn{5}{c}{ Dependent measures } \\
\cline { 2 - 7 } & \multicolumn{5}{c}{ PMC } & \multicolumn{5}{c}{ CZW } \\
\hline Factor & df & F & sig. & df & F & sig. \\
\hline Amplitude & 1,14 & 39.37 & $* *$ & 1,14 & 14.13 & $* *$ \\
Simulator & 1,14 & 3.77 & - & 1,14 & 6.33 & $*$ \\
Amplitude $\times$ Simulator & 1,14 & 1.92 & - & 1,14 & 0.67 & - \\
\hline \hline
\end{tabular}

The statistical test revealed a significant difference between the two simulators for the CZW but not for the PMC. As expected, the amplitude had a significant effect on both the PMC and CZW and there were no interaction effects between amplitude and simulator.

\section{Discussion}

Like in previous studies the perceived coherence zones were influenced by the amplitude of the visual cue and the frequency of the stimuli. However, unlike expected, at the higher amplitude and higher frequency condition, the PMC and CZW values increased with respect to the one-to-one line. This condition also presented the larger 95\% confidence interval, indicating perhaps that subjects had more difficulty or were less consistent in determining the limits of the coherence zone for this condition.

The comparison between the two frequencies in terms of acceleration did not show an increase in PMC for the conditions with lower frequency, as was hypothesized. However, when representing the conditions and respective PMC and CZW values in velocity, the expected trend is visible. For the lower frequency, the $\mathrm{PMC}$ was higher than for the higher condition. Although this is in agreement with the assumption that 
the gain imposed by the dynamics of the SCC is not taken into account during the internal comparison of the visual and inertial paths, definite conclusions can not be made, since the difference between the PMCs proved to be statistically not significant. Also interesting to note is the inverse trend of the CZW which shows a lower value for the lower frequency than for the higher frequency. It has been observed before ${ }^{18}$ that the CZW increase is roughly proportional to the increase in PMC, similar to what can be seen in the present results when PMC and CZW values are shown in acceleration space. When represented in velocity space, however, the CZW seems to be less closely related to the PMC. This result might suggest that the perceived inertial velocity amplitude is being taken into account for the internal comparison with the visual signal and the determination of a subjective one-to-one match, but that the perceived acceleration level influences how much subjects allow a deviation from that one-to-one match. One other possibility would be that for the lower frequency condition, which has also the lowest amplitude in terms of acceleration, $3 \mathrm{deg} / \mathrm{s}^{2}$, the absolute motion perception threshold is influencing the lower bound of the coherence zone. For a better insight into this effect, in Figure 7, the coherence zones are plotted in terms of upper and lower threshold for both acceleration and velocity amplitudes.

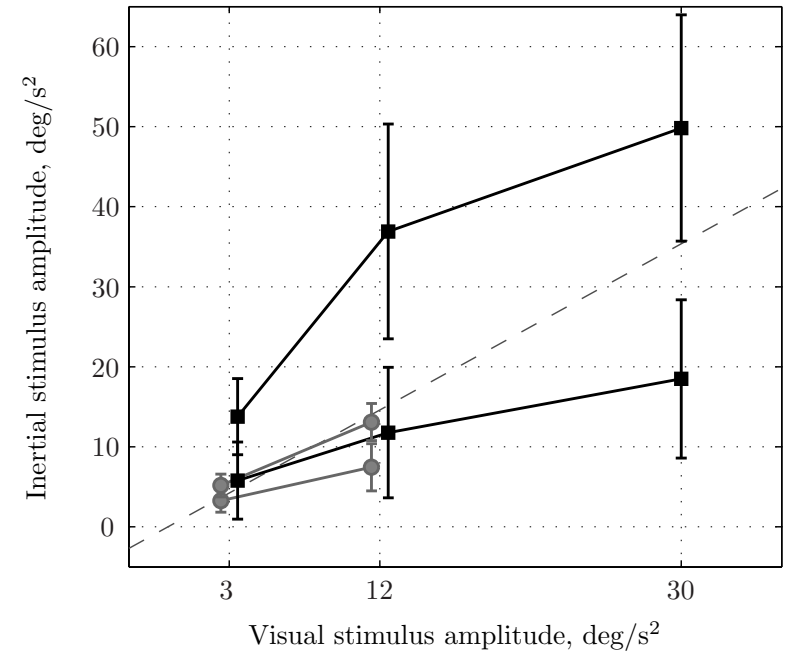

(a) Acceleration.

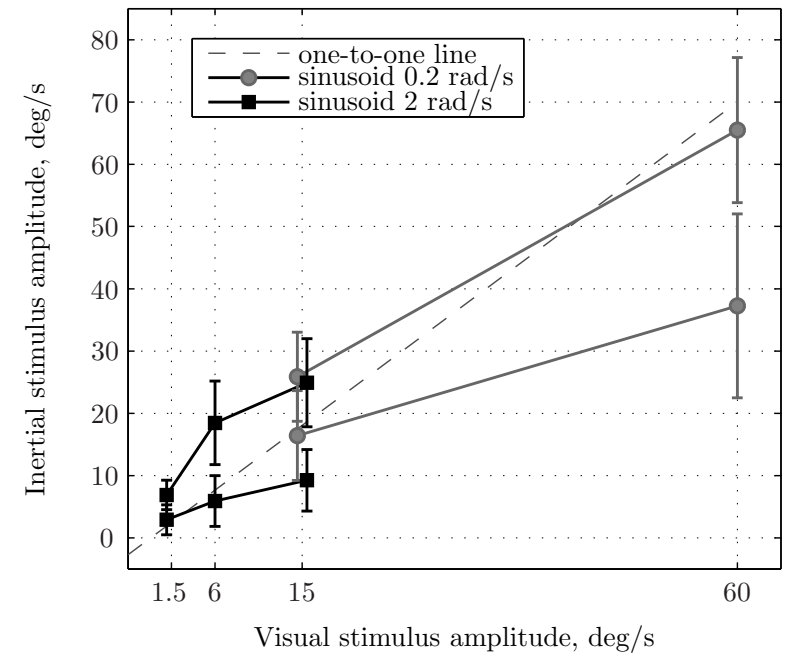

(b) Velocity.

Figure 7. Upper and lower thresholds for all conditions displayed in both acceleration and velocity units. Error bars indicate the $95 \%$ confidence interval of the means.

As can be seen in Figure 7(b), the upper thresholds for both conditions are quite similar whereas the lower thresholds differ, with the condition with the lowest frequency showing the highest value. Figure 7(a) shows that the lower threshold for the lower frequency condition approaches $3 \mathrm{deg} / \mathrm{s}^{2}$. Although this value is probably still well above the sensory threshold for this frequency, ${ }^{13,23}$ a slightly lower inertial amplitude might be too low to allow subjects to make a fair comparison with the perceived visual motion. If the lower bound of the coherence zone is in fact being influenced by the inertial sensory threshold, then, not only the decreased CZW but also the increased PMC for the lower frequency condition might be a direct consequence of this. In that case, the perceived one-to-one match might be lower than what the PMC indicates, but the upper and lower thresholds are not symmetrically distributed around the subjective one-to-one point. This would refute the hypothesis that the coherence zones are influenced by the SCC dynamics.

To avoid a biased measurement of the coherence zone lower threshold, higher acceleration amplitudes should be chosen. However, it is difficult to find a suitable velocity signal amplitude for two such different frequencies as 0.2 and $2 \mathrm{rad} / \mathrm{s}$ that still results in acceleration signals that are comfortable for the subjects and fit within the simulator's velocity and acceleration motion space. One other possibility would be to approximate the frequencies to, for example, 0.3 and $1 \mathrm{rad} / \mathrm{s}$. At closer frequencies it is easier to find an appropriate velocity amplitude, although the gains introduced by the SCC are also closer. If the gains are similar, then any existing difference between the coherence zones measured at the two frequencies will be more difficult to observe due to intra- and inter-subject variability.

The different visual scenes did not result in different PMCs or CZWs. The CZW with the star field was slightly higher, possibly indicating that there is more uncertainty in the visual estimation of self-velocity. However, without further research not much can be said about the effect of the visual scene content on the 
perception coherence zones.

The comparison of the results obtained in the Simona and in the Desdemona simulator shows a considerable difference between the two simulators. Although the values of the PMC and CZW obtained in Desdemona were much higher that the ones from Simona, statistically only the CZW values were considered to be significantly affected by the simulator factor. The data from Desdemona had also a much higher variance resulting in a larger confidence interval.

The values for the PMC found in the Desdemona simulator were considerably higher than the one-toone line, indicating an overestimation of the visually perceived self-velocity or an underestimation of the inertially perceived self-motion. As the visual display in the Desdemona simulator is much closer to the observer than in the Simona simulator, and there is no collimation, the objects in the visual scene might be perceived as closer to the observer than in Simona. If that is the case, than the displacement of these objects at a certain velocity might be interpreted as a higher self-velocity than if the objects are perceived as being further away from the observer. This overestimation of the visually perceived motion would then lead to a higher matching inertial motion, explaining the higher values found for the PMC.

Although the two visual display systems differed also in other aspects, collimation might be the one playing the most important role in the subjective estimation of self-velocity. Chung et al. ${ }^{24}$ investigated the effect of collimation, field-of-view and resolution on the pilot's control performance and subjective ratings during a hover task. They concluded that collimation had a positive influence in lateral velocity control and pilot's perceived sense of motion. A wider field-of-view showed added value for position and attitude control and resolution had no significant effect. So, whereas the field-of-view might be important for the perception of orientation with respect to the world, absence or presence of collimation seems to influence subjects perception of self-velocity and overall sense of motion.

Besides the difference in visual system, also the visual scene was slightly different, although if the results from the comparison between the star field and airport scene are to be trusted, the specific visual scene might have less influence than the visual system characteristics.

One other important difference between the two studies was the experimental method used. Both relied on a self-tuning method, although in Simona that was accomplished by letting subjects increment and decrement the inertial motion amplitude in consecutive trials, whereas in Desdemona tuning was done inthe-loop and the inertial motion gain could be changed continuously. Nevertheless, the effect of amplitude on the coherence zones showed the same trend in both simulator studies and was independent of the simulator used.

\section{Conclusions}

The comparison between the two studies in different simulators showed large differences in the measured point of mean coherence (PMC) and coherence zone width (CZW). This might seem discouraging for motion perception research in motion simulators, since results in one simulator can apparently not be easily transposed to other simulators. Nevertheless, if the focus remains on the trends rather than on the absolute values, then the conclusions drawn are similar for both studies: coherence zone widths increase with increasing visual cue amplitude, and at higher amplitudes the point of mean coherence tends to lower with respect to the on-to-one line.

The visual scene content had no influence on the measured coherence zones.

Regarding the effect of frequency, if the coherence zone measurements are observed in velocity space, then the trend of the PMC for lower stimuli frequency seems to support the hypothesis that the SCC dynamics influence the perceived coherence between visual and inertial cues. However, the values found for the CZW and the individual lower and upper thresholds indicate that there might be an effect of the inertial sensory thresholds for the condition with the lowest acceleration amplitude. For this reason the main hypothesis could not be confirmed.

For further research into this effect, care should be taken in the choice of suitable stimuli frequencies and amplitudes. At low frequencies higher acceleration profiles will have high velocity amplitudes which might be uncomfortable for subjects and/or reach simulator limits. The effect of frequency on perception coherence zones is, for this reason, easier to investigate at the middle frequency range (1 to $9 \mathrm{rad} / \mathrm{s}$ ) and high frequency range $(>9 \mathrm{rad} / \mathrm{s})$. 


\section{Acknowledgments}

The first author is supported by a Toptalent grant from The Netherlands Organisation for Scientific Research (NWO). The authors wish to thank Bruno J. Correia Grácio for helping in the design and implementation of the in-the-loop self-tuning method.

\section{References}

${ }^{1}$ van der Steen, F. A. M., Self-Motion Perception, Ph.D. thesis, Delft University of Technology, 1998.

${ }^{2}$ Young, L. R., Dichgans, J., Murphy, R., and Brandt, T., "Interaction of Optokinetic and Vestibualr Stimuli in Motion Perception," Acta Oto-Laryngologica, Vol. 76, 1973, pp. 24-31.

${ }^{3}$ Pavard, B. and Berthoz, A., "Linear Acceleration Modifies the Perceived Velocity of a Moving Visual Scene," Perception, Vol. 6, No. 5, 1977, pp. 529-540.

${ }^{4}$ Melcher, G. A. and Henn, V., "The Latency of Circular Vection During Different Accelerations of the Optokinetic Stimulus," Perception and Psychophysics, Vol. 30, No. 6, 1981, pp. 552-556.

${ }^{5}$ Zacharias, G. L. and Young, L. R., "Influence of Combined Visual and Vestibular Cues on Human Perception and Control of Horizontal Rotation," Experimental Brain Research, Vol. 41, 1981, pp. 157-171.

${ }^{6}$ Probst, T., Straube, A., and Bles, W., "Differential Effects of Ambivalent Visual-Vestibular-Somatosensory Stimulation on the Perception of Self-Motion," Behavioural Brain Research, 1985.

${ }^{7}$ Mergner, T. and Becker, W., Perception and Control of Self Motion, chap. Perception of Horizontal Self-rotation: Multisensory and Cognitive Aspects, Lawrence Erlbaum Associates, Inc., 1990.

${ }^{8}$ Wertheim, A. H., "Motion Perception During Self-Motion: The Direct Versus Inferential Controversy Revisited," Behavioral and Brain Sciences, Vol. 17, No. 2, 1994.

${ }^{9}$ Mesland, B. S., About Horizontal Self-Motion Perception ..., Ph.D. thesis, Universiteit van Utrecht, 1998.

${ }^{10}$ Zaichik, L. E., Rodchenko, V., Rufov, I. V., Yashin, Y. P., and White, A. D., "Acceleration Perception," AIAA Modeling and Simulation Technologies Conference and Exhibit, Portland, OR, USA, August 9-11, No. AIAA-99-4334, 1999, pp. 512-520.

${ }^{11}$ Rodchenko, V., Boris, S. Y., and White, A. D., "In-Flight Estimation of Pilot's Acceleration Sensitivity Thresholds," AIAA Modeling and Simulation Technologies Conference, Denver, CO, USA, August 14-17, No. AIAA-2000-4292, 2000.

${ }^{12}$ Groen, E. L., Hosman, R. J. A. W., Bos, J. E., and Dominicus, J. W., "Motion Perception Modelling in Flight Simulation," RAeS Conference, "Flight Simulation 1929-2029: A Centennial Perspective", London(UK), May 26-27, 2004.

${ }^{13}$ Valente Pais, A. R., Mulder, M., van Paassen, M. M., Wentink, M., and Groen, E. L., "Modeling Motion Perception Thresholds in Self-Motion Perception," AIAA Modeling and Simulation Technologies Conference and Exhibit, Keystone, CO, USA, August 21-24, No. AIAA-06-6626, 2006.

${ }^{14}$ Groen, E. L., Valenti Clari, M. S. V., and Hosman, R. J. A. W., "Evaluation of Perceived Motion During a Simulated Takeoff Run," Journal of Aircraft, Vol. 38, No. 4, July-August 2001.

${ }^{15}$ Fortmüller, T. and Meywerk, M., "The Influence of Yaw Movements on the Rating of the Subjective Impression of Driving," Driving Simulation Conference North America, Orlando (FL), November 2005.

${ }^{16}$ Fortmüller, T., Tomaske, W., and Meywerk, M., "The Influence of Sway Accelerations on the Perception of Yaw Movements," Driving Simulation Conference Europe, Monaco, February 2008.

${ }^{17}$ Grant, P. and Lee, P. T. S., "Motion-Visual Phase-Error Detection in a Flight Simulator," Journal of Aircraft, Vol. 44, No. 3, 2007, pp. 927-935.

${ }^{18}$ Valente Pais, A. R., van Paassen, M. M., Mulder, M., and Wentink, M., "Perception Coherence Zones in Flight Simulation," AIAA Modeling and Simulation Technologies Conference, Chicago, IL, USA, August 10-13, No. AIAA 2009-6242, 2009.

${ }^{19}$ Wentink, M., Correia Grácio, B., and Bles, W., "Frequency Dependence of Allowable Differences in Visual and Vestibular Motion Cues in a Simulator." AIAA Modeling and Simulation Technologies Conference and Exhibit, Chicago, IL, USA, August 10-13, No. AIAA-2009-6248, 2009.

${ }^{20}$ Hosman, R. J. A. W. and Van der Vaart, J. C., "Thresholds of Motion Perception and Parameters of Vestibular Models Obtained from Tests in a Motion Simulator," Tech. rep., Delft University of Technology, Delft, The Netherlands, 1980.

${ }^{21}$ Sweet, B. T. and Kaiser, M. K., "Modeling of Perception and Control Attitude with Perspective Displays," AIAA Modeling and Simulation Technologies Conference and Exhibit, San Francisco, CA, USA, August 15-18, , No. AIAA-2005-5891, 2005.

${ }^{22}$ Correia Grácio, B. J., van Paassen, M. M., Mulder, M., and Wentink, M., "Tuning of the Lateral Specific Force Gain Based on Human Motion Perception in the Desdemona Simulator," AIAA Modeling and Simulation Technologies Conference and Exhibit, Toronto, ON, Canada, August 2-5, 2010.

${ }^{23}$ Heerspink, H. M., Berkouwer, W. R., Stroosma, O., van Paassen, M. M., Mulder, M., and Mulder, J. A., "Evaluation of Vestibular Thresholds for Motion Detection in the SIMONA Research Simulator," AIAA Modeling and Simulation Technologies Conference and Exhibit, San Francisco, CA, USA, August 15-18, No. AIAA-05-6502, 2005.

${ }^{24}$ Chung, W. W. Y., Sweet, B. T., and Lewis, E., "Visual Cueing Effects Investigation for a Hover Task," AIAA Modeling and Simulation Technologies Conference and Exhibit, Austin, TX, USA, August 11-14, , No. AIAA-2003-5524, 2003. 